\title{
Microstructural Changes in a Highly Irradiated ODS Ferritic MA957 Alloy
}

\author{
Dan Edwards $^{1}$ and Mychailo Toloczko ${ }^{1}$ \\ 1. Pacific Northwest National Laboratory, Reactor Materials \& Mechanical Design Group, Richland WA \\ 99301
}

To further enable the design of nuclear fuel and duct cladding that can survive neutron doses of $>300$ displacements per atom (dpa), a variety ferritic/martensitic steels and oxide dispersion strengthened (ODS) ferritic alloys are being evaluated for their response to neutron irradiation over a range of temperatures and neutron doses. Here we report on the microstructural characterization of an ODS ferritic alloy (MA957) pressurized creep tube neutron irradiated in a fast reactor at $943 \mathrm{~K}$ to 110 dpa. MA957 alloy is an Fe-13.9 wt\% Cr with minor additions of $\mathrm{Ti}$ and yttrium as $\mathrm{Y}_{2} \mathrm{O}_{3}$. This is one of the highest irradiation doses achieved for this alloy that contained enough material to perform both mechanical property and microstructural characterization. The issue of microstructural stability of the ODS ferritic alloys under neutron irradiation remains one of the most active areas of research in the development of advanced nuclear reactors [1-4].

A JEOL 7600F FEG-SEM and a Cs probe corrected JEOL ARM200CF were used to assess the changes in the microstructure due to neutron irradiation. TEM samples were prepared in an FEI Quanta 3D FIB/SEM. Texture of the creep tube was documented via EBSD and precession electron diffraction on the TEM lamella prepared from the unirradiated archive tube and irradiated tube. The second phase particles were characterized in the JEOL ARM200CF using a combination of HAADF, STEM BF, elemental mapping using a JEOL Centurio SDD system, and a Gatan Model 965 GIF with Dual EELS capability. Dislocation imaging was performed using a combination of both conventional TEM and STEM imaging, one of the first known attempts to characterize the dislocation structures of irradiated pressurized creep tubes using aberration corrected STEM. Various combinations of convergence angles and HAADF collection angles were explored to determine the best parameters for imaging dislocations as well as the ODS particles and other second phases in the microstructure.

The microstructure of the unirradiated MA957 creep tube consists of a "bamboo" grain structure, that is, high aspect ratio grains with an average length of $\sim 10 \mu \mathrm{m}$ and a diameter of $\sim 300 \mathrm{~nm}$. The tubes possess a strong <011> texture aligned with the axis of the extruded tube. The dislocation structure is fairly dense, and forms arrays of straight line dislocations that span the narrow dimension of the grains. A moderate density $\left(\sim 10^{21}\right.$ per $\left.\mathrm{m}^{-3}\right)$ of Ti oxynitrides (Ti-N-O) were found dispersed throughout the grain structure, with a size ranging from $15-200 \mathrm{~nm}$. These particles are a separate distribution from the 1-2 nm diameter Y-Ti-O nanoxides proven to exist by atom probe tomography measurements; these latter nanoparticles are responsible for the high strengthen and microstructural stability of this alloy. Careful examination in both CTEM and STEM mode revealed that each of the Ti-N-O particles down to sizes less than $10 \mathrm{~nm}$ had an attached cavity, presumably due to a cavitation effect from extrusion at higher reductions. These cavities were often aligned at the ends of the particles in the tube axis direction.

Irradiation produced substantial changes to the Ti-N-O particles, leading to what appears to be irradiation-induced dissolution of the particles at all sizes. These changes are shown in Figure 1(a) and (b), which compares elemental maps taken from the unirradiated archive tube and the irradiated tube, 
respectively. Though not shown clearly at this magnification, the particles in Figure 1(a) all have smooth edges except where cavities are attached to the particle interfaces; the particles in Figure 1(b), taken from the irradiated sample, exhibit jagged and uneven edges when viewed at higher magnifications. The cavities observed in the unirradiated tube were not found in this irradiated tube, possibly due to be removed by radiation enhanced diffusion. Dislocation loops have formed in addition to the pre-existing dislocation structure, which appears to have been altered to produce a more tangled network than seen in the unirradiated material. Despite these changes, the overall microstructure appears to remain intact during irradiation to such a high neutron dose, however, continued irradiation may lead to additional changes if the Ti-N-O particles continue to dissolve.

\section{References:}

[1] M.B. Toloczko, et al., Journal of Nuclear Materials, Vol. 453, 2014, 323-333.

[2] G.R. Odette, JOM, 66, No. 12, 2014.

[3] S.J. Zinkle and J.T. Busby, Mater Today 12(11) (2009) pp. 12-19.

[4] C. Parish and M.K. Miller, Microsc. Microanal, 20(2) (2014) pp. 613-626.

[5] The utilized PED system was transferred from Portland State University. A portion of the research was performed using EMSL, a national scientific user facility sponsored by the DOE's Office of Biological and Environmental Research and located at PNNL.

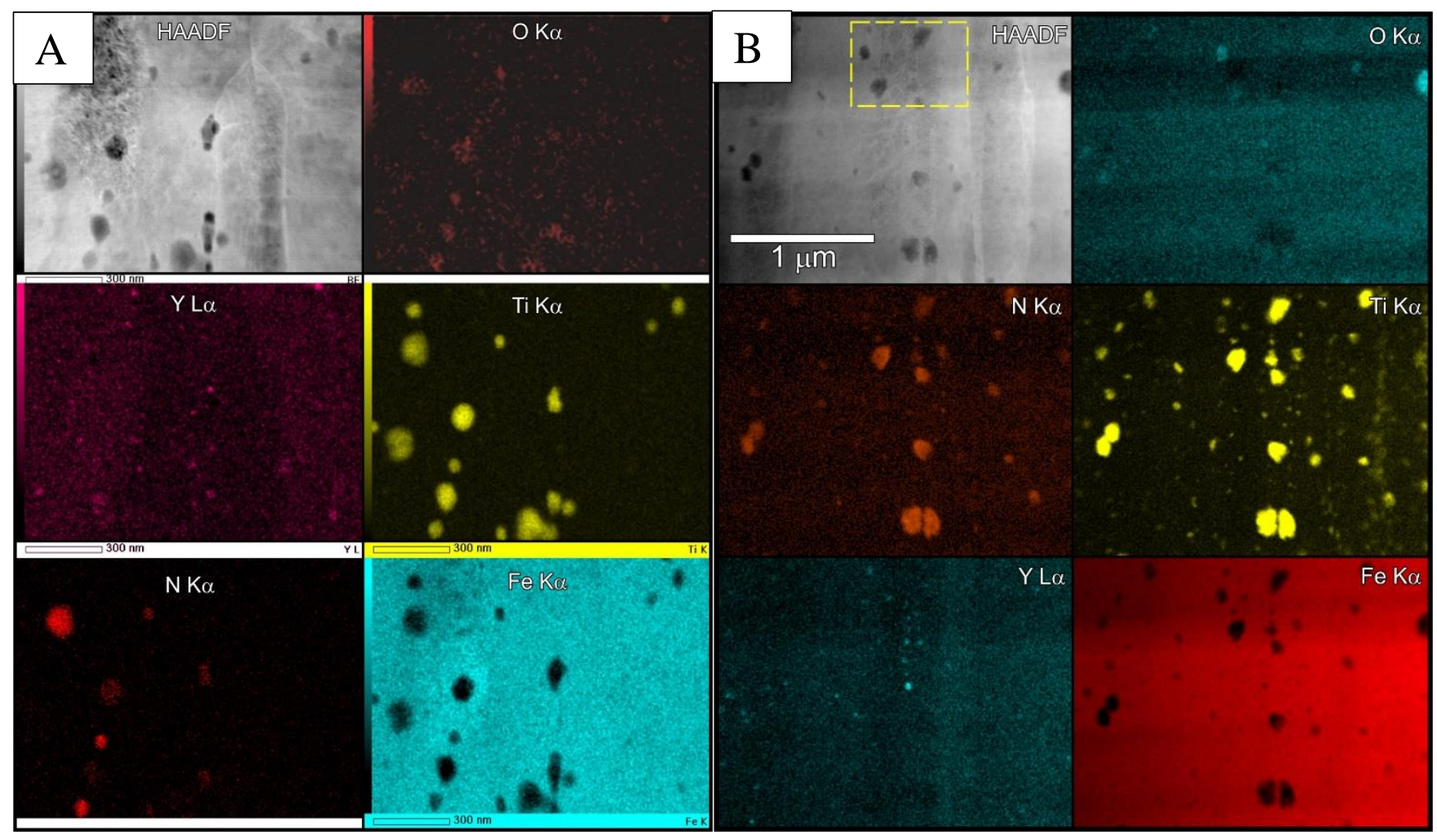

Figure 1. Elemental maps confirm the presence of a moderate density of Ti-N-O particles located in clusters and as isolated particles. The image (a) is from the unirradiated archive, in (b) from the tube irradiated at $943 \mathrm{~K}$ to $110 \mathrm{dpa}$. A low density of smaller Y-Ti-O particles are also present throughout the grains in each condition. 